\title{
Introducing our series: research synthesis and meta-research in biology
}

\author{
Shinichi Nakagawa ${ }^{1 *}$, Julia Koricheva², Malcolm Macleod ${ }^{3}$ and Wolfgang Viechtbauer ${ }^{4}$
}

\begin{abstract}
Research synthesis is the process of bringing together findings and attributes from different publications, for example, to give a more complete description of phenomena than is usually possible in a single work. We bring the Research Synthesis Series to BMC Biology to promote meta-analyses, other research syntheses including metaresearch studies, and research synthesis methodologies in biology, facilitating their dissemination to broader communities.
\end{abstract}

Keywords: Evidence synthesis, Influence synthesis, Literature synthesis, Meta-synthesis, Systematic reviews

\section{Weaving biological research together}

In this new Series, we provide a platform for publishing insights that arise from synthesizing existing research on biological topics, along with papers describing relevant methods for such synthesis and meta-research studies that elucidate biases, gaps and opportunities in the biological literature. While this is not the first contribution of BMC Biology to the topic of research synthesis [1], we strongly feel it is timely and important to establish a dedicated Series focusing on research synthesis and related methods and topics. This is because, in biology, research synthesis such as meta-analysis is, so far, primarily embraced by disciplines such as ecology, evolution and biomedical sciences, but it will certainly benefit and interest researchers in other communities in life sciences. Thus, we are truly excited to open this Series to our authors and readers.

For a biologist, although it depends on the discipline, meta-analysis is probably the most familiar type of quantitative research synthesis $[1,2]$. Some researchers, especially medical people, prefer using the terms 'systematic review and meta-analysis' or 'systematic review and quantitative synthesis' when they refer to a publication or study which includes a meta-analysis, which they consider to be simply the statistical analysis of material collected in a systematic review. Although these terms may

\footnotetext{
* Correspondence: s.nakagawa@unsw.edu.au

${ }^{1}$ Evolution \& Ecology Research Centre and School of Biological, Earth and Environmental Sciences, University of New South Wales, Sydney, NSW 2052, Australia

Full list of author information is available at the end of the article
}

be more descriptive, we feel they are a bit of a mouthful. Therefore, we use 'meta-analysis' as a shorthand for 'systematic review with quantitative synthesis' in Fig. 1, while we refer to 'systematic review with qualitative synthesis' (i.e., a review with qualitative interpretations offered) as 'meta-synthesis' [3]. Recent years have seen a marked increase in the range of approaches to systematic reviews [4], all based on a transparent, repeatable and rigorous procedure of literature search, screening and inclusion (at least in theory). This family of systematic reviews, for example, includes 'rapid reviews', a less comprehensive but quicker version of systematic reviews, and 'systematic maps' (also known as 'evidence maps'), which catalogue related studies according to their characteristics (Fig. 1).

The members of the systematic review family are often collectively termed 'evidence synthesis' because they summarize the contents of related studies providing evidence for some phenomenon of interest. Another, very different, type of synthesis analyses, for instance, bibliometric information and other alternative metrics. We refer to this type as 'influence synthesis' to distinguish it from evidence synthesis [5]. To obtain a deeper and nuanced view of the relevant literature, it has been recently proposed to combine the use of the systematic review family with bibliometrics, such as performance analysis (e.g., total citations, $h$-index) and science mapping (e.g., citation networks, co-author networks). This novel methodological framework has been called 'research weaving' [5] because it weaves different components of 


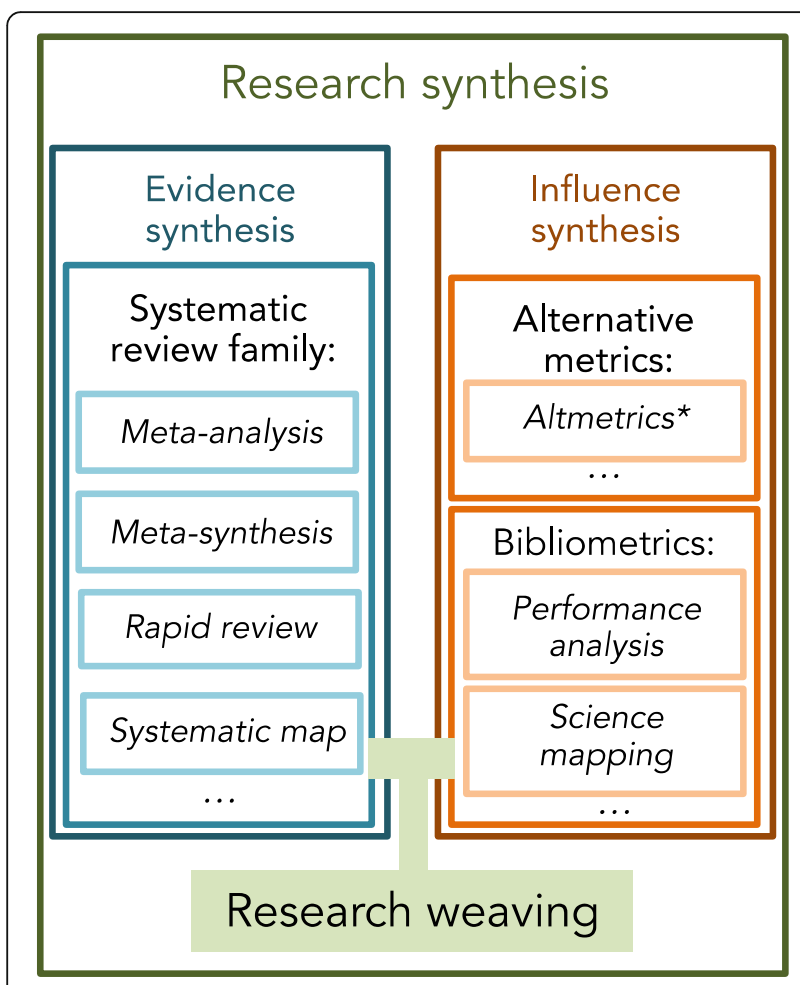

Fig. 1 Types of research synthesis and how other types of syntheses are nested within research synthesis (see the main text for more explanation). *Altmetrics is not the type of synthesis, but Altemetrics characterize social influence or impact of a publication by counting appearances in Twitter, Wikipedia, Facebook and also policy documents (https://www.altmetric.com)

research synthesis to give a richer fabric (Fig. 1). This new thematic section in BMC Biology will welcome not only any types of research syntheses, but also any associated methodologies. However, we especially welcome a synthesis or method of inter-disciplinary nature with broad significance.

\section{Methods and best practices in research synthesis in biology}

Not only is there a dramatic increase in various types of applications of research synthesis methods, the methods and practices themselves are undergoing rapid developments. Among many such methods, statistical and computational methods associated with meta-analysis comprise a field of their own. Biologists, especially ecologists and evolutionary biologists, have made significant contributions to the meta-analytic arsenal by combining phylogenetic comparative methods and meta-analysis'phylogenetic meta-analysis' where publications from different species can be aggregated in a statistically appropriate manner [1,2]. Further, meta-analytic methods relating to genome-wide association studies (GWAS) and functional magnetic resonance imaging (fMRI) are both very distinct from standard meta-analytic methodologies which conventionally use effect size statistics such as the standardized mean difference (also known as Hedges' $d$ ), the natural logarithm of odds ratio, and Fisher's $r$-to-z-transformed correlation coefficient as outcome measures [6]. The methods of synthesizing GWAS and fMRI are unique contributions of biologists (geneticists and neuroscientists), statisticians and computer scientists working together. This interdisciplinarity gives much potential to research synthesis methods in biology. Importantly, there is much more work to be done for research synthesis methods to be tailored to biologists' needs. For example, we are yet to have a meta-analytic method for integrating social network analyses, which have gained much popularity in both psychology and biology. Moreover, there is a great need to make some of the more sophisticated methods more accessible to research synthesists (e.g., via tutorials or software [7]).

We also welcome best practice and reporting guidelines for various types of research synthesis in the context of biology. Considering the significant influences of meta-analyses and other syntheses in science and beyond, it seems essential for researchers to make the quality of research syntheses consistent and high by having such guidelines. A case in point is the PRISMA (Preferred Reporting Items for Systematic Reviews and Meta-Analyses) statement. PRISMA originally aimed to improve reporting practices in meta-analyses in medical research, but is now widely used across disciplines [8]. The tens of thousands of citations they accrued over the past decade reflect their monumental impact in the community.

\section{Meta-research in biology}

Meta-research (also known as meta-science) is the study of the process of conducting research itself, using appropriate research methodology [9]. The mission of metaresearch is to provide evidence to improve scientific processes (methods, reporting, reproducibility, evaluation and incentives) by identifying and acting on research ineffectiveness, errors, biases and gaps [9]. As mentioned above, a convenient unit of research is the publication, so meta-research would often, if not almost always, use research synthesis methods, especially to obtain a bird's eye view of scientific fields. For example, a recent work has elucidated gender imbalance and gaps in scientific authorship using $>10$ million publications [10]. Notably, both methods for evidence and influence syntheses can provide such a bird's eye view [5]. Therefore, we will also consider any contributions from meta-research in biological sciences, using appropriate research synthesis methods.

Finally, it is an absolutely thrilling time to be a research synthesist with so many research articles published every day. With your ingenuity, combined with 
research synthesis methods, you can synthesize and characterize biological research regardless of the topic, from microbiota to ecosystems, or from inequality to collaboration, to bring us an even better understanding of the biological world and also the world of biologists. We hope that this thematic section will be home for many research syntheses, synthesis methods and guidelines, and also meta-research studies.

\section{Acknowledgements}

We thank the Editor-in-Chief Mirna Kvajo for excellent editing and comments in addition to supporting this new series. Losia Lagisz for commenting on the draft and helping create Fig. 1.

\section{Authors' contributions}

SN conceived the initial idea and drafted the manuscript. All authors contributed to furthering the idea and editing the manuscript; all read and approved the final manuscript.

\section{Funding}

SN was supported by ARC Discovery Grants (DP180100818 and

DP200100367).

\section{Availability of data and materials}

Not applicable.

\section{Competing interests}

The authors declare that they have no competing interests.

\section{Author details}

${ }^{1}$ Evolution \& Ecology Research Centre and School of Biological, Earth and Environmental Sciences, University of New South Wales, Sydney, NSW 2052, Australia. ${ }^{2}$ Department of Biological Sciences, Royal Holloway University of London, Egham, Surrey TW20 OEX, UK. ${ }^{3}$ Centre for Clinical Brain Sciences, University of Edinburgh, Edinburgh EH16 4SB, UK. ${ }^{4}$ Department of Psychiatry and Neuropsychology, School for Mental Health and Neuroscience, Faculty of Health, Medicine, and Life Sciences, Maastricht University, 6200 MD Maastricht, The Netherlands.

Published online: 05 March 2020

\section{References}

1. Nakagawa S, Noble DW, Senior AM, Lagisz M. Meta-evaluation of metaanalysis: ten appraisal questions for biologists. BMC Biol. 2017;15(1):18.

2. Gurevitch J, Koricheva J, Nakagawa S, Stewart G. Meta-analysis and the science of research synthesis. Nature. 2018;555(7695):175-82.

3. Siddaway AP, Wood AM, Hedges LV. How to do a systematic review: a best practice guide for conducting and reporting narrative reviews, metaanalyses, and meta-syntheses. Annu Rev Psychol. 2019;70:747-70.

4. Moher D, Stewart L, Shekelle P. All in the family: systematic reviews, rapid reviews, scoping reviews, realist reviews, and more. Syst Rev. 2015;4:183.

5. Nakagawa S, Samarasinghe G, Haddaway NR, Westgate MJ, O'Dea RE, Noble DWA, Lagisz M. Research weaving: visualizing the future of research synthesis. Trends Ecol Evol. 2019:34(3):224-38.

6. Fanelli D, Costas R, loannidis JPA. Meta-assessment of bias in science. Proc Natl Acad Sci U S A. 2017:114(14):3714-9.

7. Viechtbauer W. Conducting meta-analyses in $\mathrm{R}$ with the metafor package. J Stat Software. 2010;36(3):1-48.

8. Moher D, Liberati A, Tetzlaff J, Altman DG, Grp P. Preferred reporting items for systematic reviews and meta-analyses: the PRISMA statement. PLoS Med. 2009;6(7):e1000097.

9. Ioannidis JPA, Fanelli D, Dunne DD, Goodman SN. Meta-research: evaluation and improvement of research methods and practices. PLoS Biol. 2015; 13(10):e1002264

10. Holman L, Stuart-Fox D, Hauser CE. The gender gap in science: how long until women are equally represented? PLoS Biol. 2018;16(4):e2004956.

\section{Publisher's Note}

Springer Nature remains neutral with regard to jurisdictional claims in published maps and institutional affiliations. 